\title{
Self-Management Experiences and Challenges of Elderly Women with Type 2 Diabetes
}

\author{
Pushpamala Ramaiah Ponaiah¹, Nahla A. Tayyib², Grace Macfarland Lindsay ${ }^{3}$, Kalaiselvi Periannan ${ }^{4}$ \\ 1, 2, 3 Department of Nursing, Umm al-Qura University, Makkah, Saudi Arabia. \\ ${ }^{4}$ Department of Nursing, Oxford School of Nursing and Midwifery, UK.
}

\section{ABSTRACT}

\section{BACKGROUND}

Diabetes will occur at any age, but the presence of co-morbid conditions in the elderly population complicates its management. There are increasing concerns about managing the physical and emotional perspectives of older people whose functional and reasoning needs are on a decline. An older adult with diabetes is at increased risk of developing mental illness and is considered as one of the emerging widespread health issues of the twenty first century in India. Compliance with self-care behaviour is essential in developing a better outcome in glycaemic control and quality of life. This study aims to explore the self-care management experiences and challenges of older women with diabetes using online group discussion.

\section{METHODS}

Purposive sampling method was adopted to assign patients with diabetes from four semi-urban communities of India from different regions. Twenty-two women were employed to obtain data, and conduct group discussions a total of ten times through online sessions.

\section{RESULTS}

Three themes were generated from the collected and analysed data such as bodymind voice perception, everyday challenges, including self-management accomplishment problems in exploring novel insights of their experiences and issues of older women with regard to self-care management of diabetes.

\section{CONCLUSIONS}

Community healthcare multidisciplinary teams must be involved in providing health education to the needed population with diabetes to minimize patients' anxiety and to develop more patient focused, high quality, competent and compassionate care. In addition to monitoring patients, more attention is needed on successful adaptation to maintain a better quality of life.

\section{KEY WORDS}

Older Woman, Diabetes Mellitus, Online Focus Group, Experiences, Challenges, Quality of Life
Corresponding Author: Dr. Pushpamala Ramaiah Ponaiah, Faculty of Nursing, Umm al - Qura, University, Makkah, Saudi Arabia. E-mail: aravindanadar@gmail.com

DOI: $10.14260 /$ jemds/2020/801

How to Cite This Article:

Ponaiah PR, Tayyib NA, Lindsay GM, et al. Self-management experiences and challenges of elderly women with type 2 diabetes. J Evolution Med Dent Sci 2020;9(48):3648-3654, DOI: 10.14260/jemds/2020/801

Submission 01-07-2020,

Peer Review 14-10-2020,

Acceptance 20-10-2020,

Published 30-11-2020.

Copyright (C) 2020 JEMDS. This is an open access article distributed under Creative Commons Attribution License [Attribution 4.0 International (CC BY 4.0)] 


\section{BACKGROUND}

According to the latest 2019 data from the International Diabetes Federation, an estimated 463 million adults live with diabetes mellitus. The number will double in the year $2030 .{ }^{1}$ As of current statistics, it affects more than 62 million Indians, which is more than $7.2 \%$ of the adult population. The economic burden in India due to diabetes is among the highest in the world. ${ }^{2}$ The most common is type 2 diabetes among adults, which mostly happens when the body is resistant to insulin or does not secrete enough insulin to regulate blood sugar. In the past three decades, the predominance of the noninsulin diabetes community has risen dramatically in countries of all income groups. There is a universal effort made by leading health organizations to halt the rise in diabetes and obesity by $2025 .{ }^{3}$ Elderly adults with diabetes are more likely to end up with the risk of emerging a similar spectrum of micro vascular complications that include retinopathy, nephropathy, and foot problems. ${ }^{4}$ Diabetes mellitus impact several aspects of a person's everyday activities. ${ }^{5}$ Morbidity and mortality tend to be predominant and pose substantial healthcare burdens on both families and society level due to diabetic illness and its potential complications. India experiences an unprecedented future about the potential implications that diabetes may impose upon the nation. Several associated conditions influence the occurrence of disease throughout the country, and early identification of those factors is essential to develop optimistic change when facing health challenges across the population. ${ }^{6}$ Although Individuals with diabetes made an extensive result on the progress and development of the nature of the illness by taking part in their self-care, ${ }^{7}$ studies are reporting the global concerns consistently in terms of poor understanding of glycaemic control and self-care determination among patients with diabetes ${ }^{8}$ highlighting the necessity for self-management education and their competency in acquiring skills.

Surprisingly in India, there still a wide gap between effective diabetic self-care management practices and their implementation. There needed secondary care facilities in the appropriate, timely follow-up in carrying out investigations and screening procedures to identify the potential complications. A comprehensive diabetic care intervention package with a sound recording and cohort monitoring scheme and adequate referral strategy is needed. ${ }^{9}$ The selfmanagement technique is the crucial phase in controlling diabetes mellitus. A recent study identified how patients with diabetes adhere to coping platforms and revealed that only 35 $\%$ ( 45 - 69 years) of the researched community found to have the reasonable control of their blood glucose levels. ${ }^{10}$ Study findings demonstrated different perceptions, causes of conflict, or the female population of uncontrolled blood sugar levels that underline the necessity for better awareness to identify the body-mind status and self-care of managing patients with diabetes.

\section{METHODS}

Online focus group interview method was used to analyse older diabetic women's experiences and their challenges of sufferering from type 2 diabetes mellitus. A Focus group method of data collection will enable the researcher to facilitate a discussion among several respondents through an online platform ${ }^{11}$ that was performed between December 2019 and January 2020.

\section{Setting and Sample}

The purposive sampling technique was adopted to recruit patients with diabetic women from the different semi-urban regions of Tamil Nadu, comprising of 22 women. The criteria for inclusion were: age $\geq 60$ years, diagnosed with Type 2 diabetes for more than ten years, and volunteering to openly share their everyday experiences with the investigator and engage in an online focus group session and willing to be recorded.

\section{Ethical Consideration}

Researchers' clarified the purpose and process of this study to all women. Withdrawal policy strictly adhered to allow diabetic women to withdraw from the study at any time if they do not want to get further involved in this study. All participants rendered their consent before the focus group online discussion.

\section{Data Collection and Procedure}

The online session was conducted every four weeks that was planned tentatively, and each course duration (interview) was lasting 60 - 90 minutes. The in-depth information about the topics and activity was decided earlier to encourage the participants to share their signs and symptoms and their short term and long-term challenges. Researchers asked the participants questions such as, "In your opinion, how diabetes affected your health? "What is the preferred method of recording blood glucose reading?" "What are the strategies needed to support you in managing your blood glucose on day to day basis?" "What impact diabetes has on your day to day life?" "What type of experiences with diabetic medication usually makes people non-adherent to it," and what would you perceive as the most challenging thing about managing Diabetes?"

\section{Data Analysis}

The data was analysed by the method of thematic analysis, where the study was organized in a phased manner. The qualitative researchers must acknowledge that data analysis is conducted in a precise, consistent, and exhaustive manner through documentation, systematization, and disclosure methods of analysis with enough detail to facilitate the readers' interest and to identify whether the process is credible in order to preserve the reliability. ${ }^{12}$ According to the differences and similarities of the respondents' opinions, it was categorized under main themes and to generate subthemes. The processed data are as follows: (1) Collected data transcribed into verbatim, including recorded voices, interactive conversations; (2) The transcripts were consistently able to recognize and comprehend the statements formed by appropriate, meaningful words, signs, and symptoms related phrases, and sentences concerning both 
challenges and everyday events of sufferings; (3) The audio recorded voices from the groups were transformed into understandable words while retaining their meanings; (4) Meaning units were analysed and arranged; (5) Later correlated according to the variances and relatedness between their themes, and sub-themes were generated according to the comparability between meaning units, and (6) Sub themes with similar nature concepts were grouped to form three primary themes. ${ }^{13,14}$

Finally, all researchers held a discussion to identify whether the interpreted results were appropriate according to the recorded session, reflection dialogues, and direct conversations that had happened during online focus group discussion. Interviews during each session continued until theoretical saturation was achieved when subsequent meetings do not produce any new pieces of messages. ${ }^{15}$

Trustworthiness is the most significant appropriate criterion for evaluating the quality of the studies. Loyalty was ensured by reviewing the rigor using the following four significant criteria. 1 . Credibility: The researchers initiated a trustful relationship while the session was executed. 2 . Transferability: Debriefing was strictly followed after each session collaboratively by all interviewers to bring the real value for the collected data. 3. Dependability: Researchers had a dependable association within the team to ensure that the interview process describes sufficient detail as well to facilitate another researcher to repeat the work if the lack of information is found. 4. Conformability: Even a piece of information was not left so that complete records were preserved. The research step was documented in detail to drive as an audit trail for future research implementations, thereby confirming the accuracy of the study findings. ${ }^{16}$

\section{RESULTS}

Study participants $(\mathrm{N}=22)$. The participants comprised 22 women, and their age duration was between 60 and 75 years and followed the inclusion criteria of having included women with a history of diabetes of more than ten years. Regarding their educational status, three participants had completed primary education, six had completed high school, and the rest of them had completed senior high school education. Most participants were in the category of widowed, retired, and residing at a joint family system. The mean HbA1c level of the participant was $8.4 \%$.

\section{Physical and Mental Tiredness (Participant $=\mathbf{P}$ )}

Most of the women belong to above 63 years retired and residing with their children, they tend to execute all household activities, their physical power and stamina have become very deficient, and they are more likely to feel "physical tiredness." Responses from (P 9 \& 10): "We frequently happened to be alone because children are employed and involved with house maintenance the rest of the day, so it is normal to feel thirsty. However, when we feel powerless, we know there is some abnormal feature with our blood glucose level either higher or lower"

"Our mind is well aware, which is different from common fatigue, for the past ten years we have been suffering from

\begin{tabular}{|cc|}
\hline $\begin{array}{c}\text { Themes } \\
\text { Perception Body-Mind } \\
\text { Voices }\end{array}$ & $\begin{array}{c}\text { Physical and mental tiredness, excessive hunger and thirst, } \\
\text { frequent micturition, blurring of vision, diaphoresis, skin } \\
\text { itching: face, salt deposits on the body }\end{array}$ \\
$\begin{array}{c}\text { Hecognizing Diabetes and to adhere to dieting, recognizing the complication } \\
\text { Challenges }\end{array}$ & $\begin{array}{c}\text { such as hyperglycaemia and renal disease, comparison of } \\
\text { diaphoresis and exercise, emotions complicate self- } \\
\text { management }\end{array}$ \\
$\begin{array}{c}\text { Self-Management } \\
\text { Accomplishment Problem }\end{array}$ & $\begin{array}{c}\text { Diet dilemmas during a social event, hard to perform } \\
\text { regular exercises and maintain dieting, psychological } \\
\text { issues in conjunction with diabetes issues. }\end{array}$ \\
\hline Table 1: Summary of Findings of the Themes and Sub-Themes \\
Based on the Experiences of Diabetic Patients
\end{tabular}

diabetes we can focus our mind on any abnormal physical changes occurring to us" (P $7 \&$ 8)

\section{Excessive Hunger and Thirst}

"I am an old woman, so I feel fragile: I often feel more drained after doing even a small scale work for a few minutes, I mean, which needs to be considered as a normal task. However, when my tongue has a crazy perception of appetite or I would like to have porridge with buttermilk, I assume that my blood glucose level has reached peak level" (P 1). On the contrary with "When my blood glucose fall, I tend to consume something, and when I feel hungry that simultaneously I tremble" (P 2)

\section{Frequent Micturition}

When the patients are busy getting engaged with performing social activities, they do not have proper awareness of monitoring their level of blood glucose to quantify. Instead, they utilized their observable body cues. "If I frequently urinate beyond five times in one morning, which is the variation from what I normally do, then I will have the possibility of identifying the underlying mechanism that my blood glucose tends to be reaching abnormal." (P 4 \& 6)

\section{Blurring of Vision}

The dependent older woman can recognize physical changes in their blood sugar levels in their daily routine as a blurring vision. "I have found that I cannot perfectly look at things clearly what I used to witness very well; my glucose level increases..... I begin to experience black spots on the objects, and my blood sugar level is extremely high" (P 3)

\section{Diaphoresis \& Skin Itching}

"If I feel abnormally triggering heavy sweating even during light activities or sweating in cool temperatures, I can guess that my sugar level is abnormal." (P 5). "When my face skin becomes dry, I can conclude that I can predict my abnormal blood level sugar" (P 9).

\section{Salt Deposits on the Body}

"When I experience salty deposit all over my body I can confirm that it is because of high salt intake of food items that might increase my blood pressure" (P 10)

\section{Recognizing Diabetes and Challenges}

Self-care management game plan for diabetes women in online discussions and the dialogue included hard to adhere to 
dieting, recognizing the complication such as hyperglycaemia and renal disease.

A comparison of diaphoresis, exercise, and emotions do complicate self-management. The women have contributed several opinions, challenges, and suggestions similarly tried to acknowledge diabetes and its management from entirely different aspects.

In India, the economic impact and sensitivity towards food consumption, particularly salient for older women since they are more likely to hail from low-income groups due to retirement, dependency lifestyle, and out-living savings. "I am a hard strived woman in my career, and I saved enough money to look after myself after retirement. However, I cannot enjoy a healthy lifestyle after retirement with my savings due to family financial commitment" (P 11).

\section{Contributions from Online Group Discussion}

"I would hardly follow diet control because it does not seem that I have time to take special precautions to strictly maintain my diet. Hence I eat whatever is available" (Participant 14). As of concern, "To maintain a healthy practice, all people have to follow diet control, regardless of physical illness and financial crisis, and the family system needs to adhere to diet control" (P 12) strictly.

From the online group discussion, women learned to adapt to the perfect lifestyle amid any coexistent situation. "Regardless of the family system, it is the responsibility of each individual to manage with the available food items to follow dieting" (P 13).

\section{Recognising Complications Such as Hyperglycaemia and Renal Disease}

"I heard drugs are the ones causing side-effects because it is a chemical, rather I could rely more on alternative medicines such as a combination of cumin seed, flaxseed, fenugreek, black cumin, mango seed, and Tanner's cassia...," (P 14).

In Indian culture, diabetic medications are considered by rural women as poisonous, chemical compounds, heat producing elements, and cause renal damage. "I also used to minimize the number of doses most of the days, and consume the regular dose only at the time of special occasions or events when I eat delicious food," (P 15).

\section{Comparison of Diaphoresis and Exercise}

Exercise takes part in a crucial role in regulating glucose in the blood for a diabetic person. In the online group discussion, women shared their routine about walking, yoga, and breathing exercises and the effect of these activities on controlling blood sugar. "Since from the beginning, I have been doing yoga in the mornings of every day by watching media because I feel healthy, and I am confident that it can help to reduce my blood sugar level" (P 16). "I am aware of the benefits of doing regular exercises; however, I get knee joint pain while doing it. So I deliberately restrict in doing exercises 3 - 4 days in a week" (P 17). "Perspirations can make my blood sugar to consume because I am old age group I cannot make it out by doing the one which will make me perspire more, rather I am happy doing morning yogas," (P 22).

\section{Emotions Complicating Self-Management}

In group sharing, they shared their mindful activities to get rid of their piled up psychological stress. "When I realized that stress is the pivotal causative factor in raising my blood sugar level, I usually try to get diversion of my mind by some breathing exercises and meditation" (P 8). "I know very well that I cannot control my emotions; however, I would like to share with everyone that, joy, happiness, and pleasure giving activities indeed determines your emotions" (P 18). "Sometimes, when I am upset, I regret that I do not have the liberty to consume my desired dishes. Such a moment will tempt me to eat more sweet recipes" (P 19).

\section{Diet Dilemmas during a Social Event}

During social events in Indian culture, women face quite a lot of challenges; diabetic women usually tend to go into guilt and depression if they do not strictly control their mind on avoiding an unhealthy diet. "If I do not attend social occasions, my family friends will feel rejected, and I do not give them significant importance; however, I feel more guilty, depressed, and loss of self-control" (P 20).

\section{Hard to Perform Regular Exercises and Maintain Dieting}

"Doing is ever better than knowing, but knowing is easier than doing, I cannot put it in effect as a routine of my life. No one prefers to lead an unhealthy life experiencing signs and symptoms of hyperglycaemia, and with all high-risk co-morbid conditions" (P 21).

\section{Psychological Issues in Conjunction with Diabetes}

Lifestyle activities can help maintain blood sugar levels in persons with diabetes, with such a healthy lifestyle, including increased exercises and a well-balanced diet that has no sugars, reduced fats, and fresh fruits and vegetables. The lifelong self-care management and the level of acceptance of strict adherence to the regulations place a substantial mental burden among old age women with diabetes. "In the morning, I am not surprised, and wondered about having my morning tiffin with less quantity at least because I cannot prevent my dizziness unless I have it, then I will have to take my medications. Later I will never be able to do exercises with a full stomach. Such contrary coexistence situations usually make me more depressed". (P 7 \& 22)

\section{DISCUSSION}

This quantitative study adopted to identify the real experiences, challenges, and shared opinions on preventive health promotion strategies of diabetic women of rural regions from Tamil Nadu. The COVID-19 pandemic situation has made them undergo unique life experiences, which was useful to develop a better approach to learn coping strategies in looking after their health. The reported physical and mental symptoms adopted the classification of generated sub-themes, which has 
precisely discussed with their observed physical perception during their daily activities.

\section{Diabetes Self-Care Management and Symptom Recognition}

The management of type 2 diabetes has undergone a drastic change with the establishment of numerous novel classes of glucose lowering remedies. This increase in diabetes therapy options represents both an opportunity, with the development of the tool kit, to optimize treatment mode and the uncertainty as to deciding on the appropriate treatment interventions punctually. ${ }^{17}$ Chronic conditions with diabetes are estimated to reach even further in the following decades because of an aging community and the adoption of unhealthy lifestyle practices. ${ }^{18}$ In diabetic patients, depression remains underdiagnosed, and it is an essential aspect of the health care provider's responsibility. The psychological burden plays a vital role in triggering anxiety and depression. Depression has a higher prevalence and could be due to an unfavourable lifestyle, and such as physical inactivity, unhealthy diet, or a stressful lifestyle. A multidisciplinary approach for the diabetic patient would help improve the outcomes of disease, decrease the number of DALYs (Disability-Adjusted Life Year), and even mortality. ${ }^{19}$ The recognized gaps in diabetes selfmanagement skills included the ability to: identify and cope with the impact of stress on diabetes, employ planning to manage hypoglycaemia, and analysing the patterns of blood glucose levels. ${ }^{20}$ In this research, diabetic older women assessed their level of blood glucose only after their perception of associated bodily symptoms with the blood glucose level. In online focus group sessions, mothers of different ages (elderly) and with the different family systems shared how they usually recognize their body and mental changes according to the different nature of perceptions. Addressing the aspects of physical, cognitive, behavioural, and social factors would help to challenge the psychological barriers associated with consistent healthy practices in managing self-care: the latter being the ultimate goal of the management of patients with diabetes. ${ }^{21}$ The health care professional can help diabetic patients to overcome the barriers to self-management, such as low-level blood sugar knowledge, lack of family support, increased family demand, and negative perception of time. The main obstacles related to foot care measures need priority focus among the diabetic group in early diagnosis and preventive model of illness. ${ }^{22}$

\section{Challenges of Diabetic Women and Healthcare Professionals}

According to the participants' shared conversation, they are aware of the importance of diet, exercise, adherence to medication, and other associated physical symptoms in recognizing the need to promote and maintain their health. However, engaging in mind dilemmas about self-care management prevents the patient from getting involved in following appropriate strategies.

The challenges in diabetes self-care management fall within two contexts: health care centers and community. Although there are many problems in meeting out the challenges, opportunities for improvement are still available. The shared responsibility of the primary health centers and sub-centers and the community is essential in preventing complications from developing the quality of life of the diabetic group. ${ }^{23}$ A study in India reported from survey allies with our findings that among people with diabetes (31\%) revealed that diabetic patients who have better diet control practice and self-care monitoring of activities were only deplorable. ${ }^{24}$ Another study had similar results that only 27.75 $\%$ of the diabetic patients engaged in walking, $24.25 \%$ had appropriate foot care, and blood glucose monitoring by 24.75 $\% .{ }^{25}$ Hence, health care organizations should focus on custom designed protection and standard programs tailored for female group older women based on national and regional attitudes on delivering the needed care with the available support systems as well as the cultural based intervention that is essential to be implemented. Gender differences and inequalities identified about causes and consequences of diabetes. Besides, in order to forecast the aetiology of observed gender variations of diabetes related complications, the associated hormonal fluctuations and socio-cultural diversity issues must be explored and considered. ${ }^{26}$ India is dubious, considered as the diabetes capital of the world as millions in the country suffer from this disease or condition. The most common myths about diabetes among women is that they should follow only a rice-free diet. Nevertheless, what actually needs to be done is, people need to adopt a balanced diet, which includes rice as a primary source of carbohydrate in moderate amounts. Although alternate sources of carbohydrates like brown rice, wheat, and millets tend to have better glycaemic control, they should not be treated as "holy foods" and rice as "unholy" one. ${ }^{27}$

\section{Self-Management Accomplishment Problem}

Several studies on exercise linked with diabetes have mainly utilized an exercise intervention to enhance the interest of patients' performing exercises. Performing physical exercises are the most effective and economic strategy in controlling diabetes, and it can also delay the incidence of potentially lifethreatening issues. The study participant agreed that performing regular physical activities would regulate blood sugar; however, maintaining this as a habit is the tricky part of their life.

Safe exercise involvement can be complicated by the occurrence of diabetes related health complications such as cardiovascular disease, nerves related disorder, or vascular changes. ${ }^{28}$ For individuals desiring to involve in low level physical activity such as small indoor steps walk, health care professionals need to practice clinical judgment in deciding whether to suggest pre-exercise testing. ${ }^{29}$ The available literature on the community based program suggests proof that lifestyle changes with physical activity can alter the incidence of diabetes or one of the type 2 diabetes mellitus risk factors and may positively affect anthropometric indices and HbA1c. ${ }^{30}$ The present study suggests that during the regular days at home, regardless of the employed or non-employed women, they felt the necessity of increased health related plan of action, which plays a vital role in lifestyle modification.

Diabetes in older adults has been linked with an escalated risk of psychological disorders and accompanying poor health in terms of quality of life in that has a more significant negative impact on all domains of lifestyle. Thus, patients with diabetes require access to mental health services in addition to 
governing metabolic abnormalities. Excessive worrying about potential risk factors of diabetes can lead to anxiety. ${ }^{31}$ The enormous increase in the risk of co-morbid depression among diabetic patients attribute to the psychological burden of disease, lack of social support, low awareness about having a chronic illness, related complications, disabilities, and the considerable mental distress. ${ }^{32}$ Notably, women (older) with diabetes are likely to have a higher prevalence of depression. ${ }^{33}$ Therefore, community health professionals should render significant attention to older people in managing their mental health rather than focusing on the physiological disease. Listening to the sufferers when they express their self-care management barriers is also essential to support and develop them to maintain and promote their healthy lifestyles.

Furthermore, the community health care team should include consultants to deal with diabetic patients who suffer from psychological issues. They need to establish a behavioral, supportive group to manage the high-risk group in adopting more positive attitudes by encouraging them to take a positive approach to life and co-exist with both physical and mental illness. Considering the small sample size and addressed the women from one state of the country, results will not be implacable for the diverse socio-cultural background of diabetic groups. Also, the recorded session of the online focus group has the drawback of limited course discussion. Hence it may not precisely contemplate changes to an individual's perspective over a long period.

\section{CONCLUSIONS}

Older women with diabetes mellitus from rural regions of Tamil Nadu identified deeper insights into the experiences, challenges, and suggestions regarding their self-care management of diabetes. The alarming core demand from supportive family systems and community health care teams necessitate a collaborative role in promoting self-care management education for women with diabetes. Health education strategy needs to adhere to appropriately intervene in the platform of early diagnosis and treatment that would prevent them from stress, anxiety, and frustration regarding their level of glucose and physical symptom. This could result in preventing diabetic patients from life-threatening difficulties.

Data sharing statement provided by the authors is available with the full text of this article at jemds.com.

Financial or other competing interests: None.

Disclosure forms provided by the authors are available with the full text of this article at jemds.com.

The authors thank all the participants for sharing their experiences and challenges through online focus group sessions.

\section{REFERENCES}

[1] International Diabetes Federation. IDF diabetes atlas. $9^{\text {th }}$ edn. Brussels, Belgium 2019. https://www.diabetesatlas.org/en/

[2] Diabetes can be controlled in 80 percent of cases in India IANS. News.biharprabha.com. 2016. Retrieved 25 May 2020.
[3] World Health Organization. Global report on diabetes. Geneva: World Health Organization 2019.

[4] American Diabetes Association. Standards Medical Care in Diabetes 2018;41(Suppl 1):119-25.

[5] Chrvala CA, sherr D, Lipman RD. Diabetes selfmanagement for adults with type 2 diabetes mellitus: a systematic review of the effect on glycemic control. Patient Educ Couns 2016;99(6):926-43.

[6] Natarajan MSS, Lakshmanan A, Ramraj B. Awareness of diabetes and its complicaions among diabetes patients and their caretakers attending a tertiary care hospital in Chennai suburban area. J Evolution Med Dent Sci 2019;8(23):1839-42.

[7] Shrivastava SR, Shriastava PS, Ramasamy J. Role of selfcare in management of diabetes mellitus. J Diabetes Metab Disord 2013;12(1):14.

[8] Dedefo MG, Ejeta BM, Wakjira GB, et al. Self - care practices regarding diabetes among diabetic patients in West Ethiopia. BMC Res Notes 2019;12(1):212.

[9] Tripathy JP, Sagili KD, Kathirvel S, et al. Diabetes care in public health facilities in India: a situational analysis using a mixed methods approach. Diabetes Metab Syndr Obes 2019;12:1189-99.

[10] Tripathy JP, Thakur JS, Jeet G, et al. Prevalence and risk factors of diabetes in a large community-based study in North India: results from a STEPS survey in Punjab, India. Diabetol Metab Syndr 2017;9:8.

[11] B2B International a gyro: company. https://www.b2binternational.com/research/methods.

[12] Nowell LS, Norris JM, White DE, et al. Thematic analysis: striving to meet the trustworthiness criteria. International Journal of Qualitative Methods 2017;16(1).

[13] Liang SY, Chuang YH, Wu SF. Preliminary application of content analysis to qualitative nursing data. $\mathrm{Hu} \mathrm{Li} \mathrm{Za} \mathrm{Zhi}$ 2012;59(5):84-90.

[14] Graneheim UH, Lundman B. Qualitative content analysis in nursing research concepts, procedures and measures to achieve trustworthiness. Nurse Educ Today 2004;24(2):105-12.

[15] Wu FL, Tai HC, Sun JC. Self-management experience of middle-aged and older adults with type 2 diabetes: a qualitative study. Asian Nurs Res 2019;13(3):209-15.

[16] Maher C, Hadfield M, Hutchings M, et al. Ensuring rigor in qualitative data analysis: a design research approach to coding combining NVivo with traditional material methods. International Journal of Qualitative Methods 2018;17:1-13.

[17] Zinman B, Skyler JS, Riddle MC, et al. Diabetes research and care through the ages. Diabetes Care 2017;40(10):1302-13.

[18] Grilo AM, Santos MC, Gomes AI, et al. Promoting patient centered care in chronic disease. Chap- 3. In: Sayligil 0, ed. Patient centered medicine. Zagreb: InTech 2017.

[19] Badesu SV, Tataru C, Kobylinska L, et al. The association between diabetes mellitus and depression. J Med Life 2016:9(2):120-5.

[20] Adu MD, Malabu UH, Malalu-Aduli AEO, et al. Enablers and barriers to effective diabetes self- management: a multi-national investigation. PLoS One 2019;14(6):e0217771. 
[21] Kalra S, Jena BN, Yeravdekar R. Emotional and psychologican needs of people with diabetes. Indian J Endocrinol Metab 2018;22(5):696-704.

[22] Taksande BA, Thote M, Jajoo UN. Knowledge, attitude and practice of foot care in patients with diabetes at central rural India. J Family Med Prim Care 2017;6(2):284-7.

[23] Al-Alawi K, Al Mandhari A, Johansson H. Care providers' perceptions towards challenges and opportunities for service improvement at diabetes management clinics in public primary health care in Muscat, Oman: a qualitative study. BMC Health Serv Res 2019;19(1):18.

[24] Mohandas A, Bhasin SA, Upadhyay M, et al. Diabetes self care activities among adults 20 years and above residing in a resettlement colony in East Delhi. Indian J Public Health 2018;62(2):104-10.

[25] Srinath KM, Basavegowda M, Tharuni NS. Diabetic self care practices in rural Mysuru, Southern Karnataka, India - a need for diabetes self- management educational (DSME) program. Diabetes Metab Syndr 2017;11 Suppl 1:S181-6.

[26] Bajaj S, Jawad F, Islam N, et al. South Asian women with diabetes: psychosocial challenges and management: consensus statement. Indian J Endocrinol Metab 2013;17(4):548-62.
[27] The Indian Express, Retrieved on 10th June, 2020. https://indianexpress.com/article/lifestyle/health/myt hs-diabetes-harmful-misleading

[28] Sigal RJ, Kenny GP, Wasserman DH, et al. Physical activity/ exercise and type 2 diabetes. Diabetes Care 2004;27(10):2518-39.

[29] American Diabetes Association. Physical activity/exercise and diabetes. Diabetes Care 2004;27(Suppl 1):S58-62.

[30] Shirinzadeh M, Afshin-Pour B, Angeles R, et al. The effect of community- based programs on diabetes prevention in low and middle-income countries: a systematic review and meta-analysis. Global Health 2019;15(1):10.

[31] Bansal R, Chatterjee P, Chakravarty A, et al. Diabetes: a risk factor for poor mental health in ageing population. J Geriatric Mental Health 2018;5(2):152-8.

[32] Khaledi M, Haghighatdoost F, Feizi A, et al. The prevalence of comorbid depression in patients with type 2 diabetes: an updated systematic review and meta-analysis on huge number of observational studies. Acta Diabetol 2019;56(6):631-50.

[33] Chen G, Wu Y, Wang T, et al. Association between serum endogenous secreatory receptors for advanced end products and risk of type 2 diabetes mellitus with combined depression in the Chinese population. Diabetes Technol Ther 2012;14(10):936-42. 